\title{
Sleep-Related Attentional Bias to Word Stimuli in Patients with Insomnia Disorder
}

\author{
Soohyun Kim', Seong min Oh², Jeong Eun Jeon², Ha Young Lee², Inkyung Park², \\ Juhyun Park', Mi hyun Lee², and Yu Jin Lee \\ 'Department of Neurology, Gangneung Asan Hospital, Gangneung, Korea \\ 2Department of Psychiatry and Center for Sleep and Chronobiology, Seoul National University College of Medicine, \\ Seoul National University Hospital, Seoul, Korea \\ ${ }^{3}$ Department of Psychological and Brain Sciences, University of lowa, lowa, IA, USA \\ ${ }^{4}$ Department of Psychology, University at Buffalo, Buffalo, NY, USA
}

\begin{abstract}
Objective: This study investigated sleep-related attentional bias toward word stimuli in patients with insomnia. In addition, by diversifying the word stimuli, we investigated bias towards various words by insomnia patients. Methods: A total of 24 insomnia patients based on the Diagnostic and Statistical Manual of Mental Disorders, fifth edition (DSM-5) criteria and 21 healthy controls were enrolled. Two types of words, including 39 sleep-related and 40 neutral words, were used as experimental stimuli. In the subjective emotional rating task, all participants were asked to rate the emotional intensity of each word on a 7-point Likert scale $(-3=$ most negative and $+3=$ most positive). Subsequently, participants were asked to indicate whether each word stimulus was associated with sleep. Results: There were no significant differences in selfrated valence on the types of words between groups. However, there were significant differences in the ratio of 'sleep-relatedness' responses and whether each stimulus was associated with sleep in the neutral category $(p<0.05)$. Insomnia patients responded to neutral stimuli as sleeprelated more frequently than the control group. Conclusion: Our results support a sleep-related attentional bias towards neutral stimuli among insomnia patients. This interpretive bias may contribute to the underlying mechanism of chronic insomnia.
\end{abstract}

Key Words: Insomnia; Sleep-related attentional bias; Interpretive bias; Word stimulus

Received: January 28, 2019 Revised: April 21, 2019 Accepted: April 30, 2019

Corresponding author: Yu Jin Lee, MD, PhD, Department of Psychiatry and Center for Sleep and Chronobiology, Seoul National University College of Medicine, Seoul National University Hospital, 101 Daehak-ro, Jongno-gu, Seoul 03080, Korea.

Tel: 82-2-2072-2456, Fax: 82-2-744-7241, E-mail: ewpsyche@hanmail.net

(a) This is an Open Access article distributed under the terms of the Creative Commons Attribution Non-Commercial License (https://creativecommons.org/licenses/bync/4.0) which permits unrestricted non-commercial use, distribution, and reproduction in any medium, provided the original work is properly cited.

\section{INTRODUCTION}

Insomnia is one of the most common sleep disorders. About onethird of the general population complains of insomnia symptoms at least once in their life [1]. Insomnia disorder is defined as persistent symptoms such as difficulty initiating sleep, difficulty maintaining sleep, or early morning awakening with the inability to return to sleep at least 3 nights per week for at least 3 months based on the Diagnostic and Statistical Manual of Mental Disorders, fifth edition (DSM-5) [2]. These sleep disturbances cause daily dysfunction and can lead to serious cognitive, emotional, or even physical problems. Patients with insomnia show cognitive dysfunction in various areas such as memory, executive function, and attention [3], and have a high chance of developing psychiatric disorders such as depression and anxiety [4,5]. Furthermore, a relationship between chronic insomnia and cardiometabolic problems such as hypertension $[6,7]$ and diabetes $[8,9]$ has been reported. These adverse impacts can result in an economic burden to both the individual and society. Therefore, it is important to understand the features of insomnia and management of the symptoms.

One interesting feature of chronic insomnia is that maladaptive thoughts and behaviors can be associated with developing or maintaining insomnia [10,11]. In transient or situational insomnia, the symptoms may improve after the stressful situation has resolved. Otherwise, short-term sleeping pills can be helpful for insomnia symptoms. However, patients with persistent insomnia often have excessive worry and misperceptions about sleep [12]. They tend to focus on their ability to control their sleep and believe that they cannot function if they do not sleep. These maladaptive beliefs about sleep can result in maladaptive behavior, ultimately leading 
to chronic insomnia. For example, insomnia patients go to bed early and lay in bed too long even though they are not tired, or they sleep during the day to compensate for the lack of night sleep. With increased worry about sleep, sleep can become more interrupted; this cycle causes insomnia to develop and persist $[11,13]$.

A prominent model to explain maladaptive processes in patients with chronic insomnia is that they have biased attention to sleeprelated stimuli. Harvey [13] proposed a cognitive model for chronic insomnia. In this model, excessive negatively biased cognitive activity (e.g., excessive worry or catastrophic thoughts resulting from the lack of sleep) leads to autonomic hyperarousal and emotional distress that triggers an anxious state to prepare for fight or flight. Thus, insomnia patients become more sensitive to internal (e.g., heart rate) and external (e.g., noise) cues that can threaten sleep. An aroused state and selective attention to sleep-related stimuli can cause the patients to overestimate cues, resulting in maladaptive safety behaviors. Through this cognitive process, excessive negatively biased cognitive activity can be reinforced.

Previous studies using cognitive tasks have supported sleep-related attentional bias in insomnia patients; the most common is the emotional Stroop task [14]. In this task, response latency can be calculated as an interference index by subtracting reaction times to a neutral stimulus from reaction times to an emotional stimulus. In the sleep-related emotional Stroop task, insomnia patients showed a higher interference index score compared with controls [15-17]. Thus, insomnia patients are more attuned to sleep-related words and require more cognitive load to react.

However, there have been controversial results in other studies. The first study using the sleep-related emotional Stroop task showed no significant interference effect from sleep-related words [18]. Sagaspe et al. [19] hypothesized that heightened anxiety levels from sleep deprivation would trigger the interference effect, but this was not observed. Furthermore, Spiegelhalder et al. [15,16] showed attentional bias in insomnia patients, but this was not replicated in later work [20]. Meanwhile, Barclay and Ellis [21] showed an interference effect based on negative words rather than sleeprelated words, and Zhou et al. [22] reported a significant interference effect from sleep-related positive words and a marginally significant effect from sleep-related negative words.

These inconsistent results can be reproduced using differing approaches even though researchers used the same emotional Stroop task paradigm. We attempted to determine the reason for the inconsistent results on the emotional Stroop task by understanding the attentional bias toward the word stimuli. We wanted to exclude the elements of the Stroop task and focused on how patients perceived the words themselves. By identifying how different insomnia patients perceive the word stimulus, we can increase our understanding of sleep-related bias. We identified the bias in two different manners: first, we examined how different insomnia patients scored word with emotional valence; and second, we examined whether they associated the words with sleep. In addition, by examining both sleep-related and neutral words, we investigated insomnia patients' cognitive bias toward two types of words.

\section{METHODS}

\section{Participants}

A total of 29 patients with insomnia disorder based on DSM-5 criteria were enrolled at the outpatient clinic of the Department of Psychiatry at Seoul National University Hospital. We excluded patients with serious neurological or psychiatric disorders by performing psychiatric interviews in the outpatient clinic. Next, 22 healthy controls were enrolled via an advertisement. Five insomnia patients and one healthy control were excluded because of missing data in both emotional valence and sleep relevance items (three insomnia patients and one healthy control) and a lack of demographic information (two insomnia patients). Thus, 24 insomnia patients ( 13 females and 11 males; average age $=43.625 \pm$ 12.846 years) and 21 healthy controls ( 14 females and 7 males; average age $=32.857 \pm 8.338$ years) were included in the analysis. The study was approved by the Institutional Review Board of Seoul National University Hospital (IRB No. H-1603-073-748). Prior to study initiation, a complete description of the study was given and written informed consent was obtained from the participants.

\section{Questionnaire}

We made our own questionnaire to assess bias towards word stimuli. Two types of words, including sleep-related and neutral words, were used as experimental stimuli. The sleep-related words were determined from past studies on attentional bias to sleep-related words with a sleep-related emotional Stroop task $[15,16,19,21,23]$. We translated the sleep-related words into Korean and reviewed the words with sleep experts. Based on this review, several words were excluded because the words had a similar meaning in Korean (e.g., bushed and exhausted) or the words were not translated to one word (e.g., stretched out and night reveler). Finally, 39 sleep-related words were included in the study. In total, 40 neutral words were randomly selected from the word set of the Korean version of the California Verbal Learning Test. We calculated the frequency of the words [24] and found no significant differences in the mean use frequency (sleep-related word $=153.308 \pm 288.014$, neutral word $=66.000 \pm 81.859$ ). There was also no significant difference in the mean number of syllables (sleep-related word $=2.539 \pm 0.790$, neutral word $=2.275 \pm 0.126$ ) between word types.

The questionnaire was designed to address the subjective emotional valence and sleep-relatedness of each word. In the items to rate subjective emotional valence, all participants were asked to rate the emotional valence of randomly presented words on a 7point Likert scale ( -3 was the most negative and +3 was the most positive). Subsequently, participants were asked to indicate whether each word stimulus was associated with sleep.

\section{Analysis}

First, a two-way repeated measure analysis of variance (ANOVA) (group $\times$ type of word) was performed to compare the subjective emotional word valence according to groups and types of 
words. Second, another two-way repeated measure ANOVA was performed for the ratio of 'sleep-relatedness' responses with the same factors (group $\times$ type of word). We calculated the ratio of 'sleep-relatedness' responses for each type of word to change the categorical variable to a continuous variable. Only 19 insomnia patients were included in this analysis because the other 5 patients had missing items. Third, the chi-square test was performed for categorical responses of each word stimulus to determine which words were considered sleep-related between the two groups. Data were analyzed using PASW Statistics for Windows (Version 18.0; SPSS Inc., Chicago, IL, USA) with statistical significance set at $\mathrm{p}<0.05$.

\section{RESULTS}

There were no significant differences in subjective emotional valence to the two types of words between the two groups (Figure 1 ). We calculated the mean and standard deviation of the valence

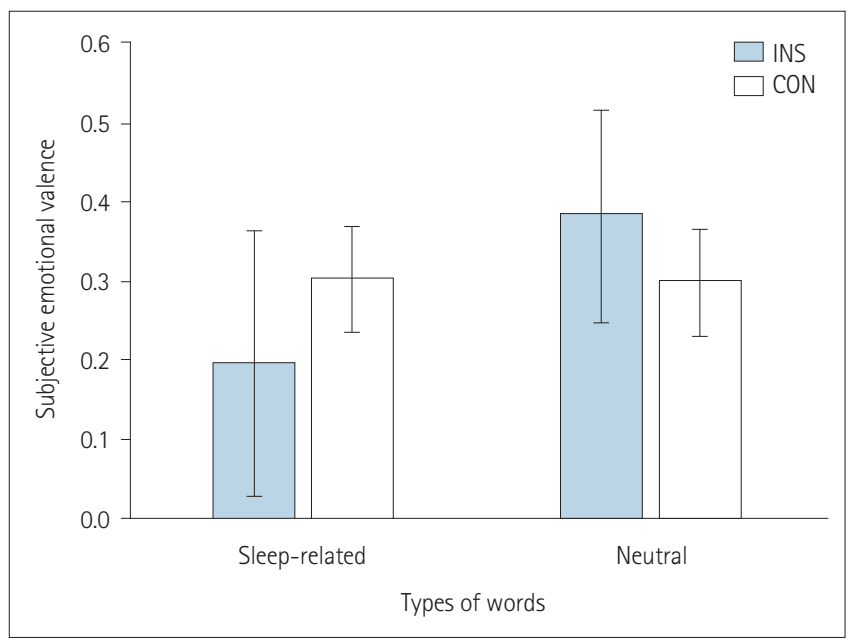

Figure 1. Subjective emotional valence. There were no differences in word valence according to types of words and groups. INS: insomnia patients, CON: healthy control.

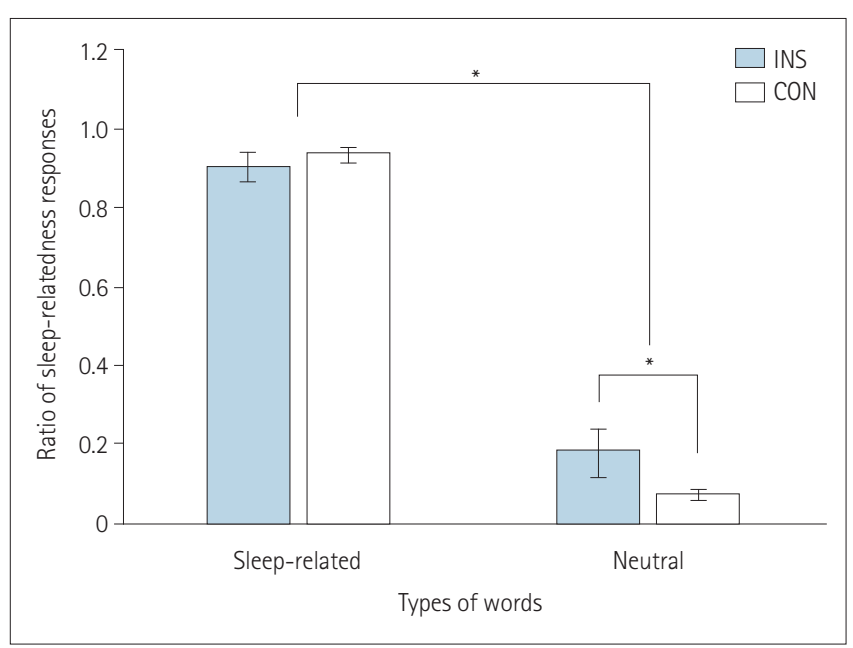

Figure 2. The ratio of 'sleep-relatedness' responses according to types of words and groups. Insomnia patients responded to neutral words more commonly as sleep-related $\left({ }^{*} p<0.05\right)$. INS: insomnia patients, CON: healthy control. in each condition as follows: sleep-related word, insomnia patients (INS) $0.195 \pm 0.823$ and healthy controls (CON) $0.302 \pm 0.309$; neutral word, INS $0.382 \pm 0.665$ and CON $0.299 \pm 0.312$.

There was a significant interaction in the ratio of sleep-relatedness responses to the two types of words between the two groups $\left(\mathrm{F}_{[1,38]}=4.263, \mathrm{p}<0.05\right)$ (Figure 2). The INS group responded more frequently to neutral stimuli as sleep-related stimuli. We calculated the mean and standard deviation of the ratio of 'sleep-related-

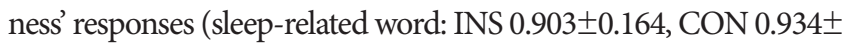
0.092; neutral word: INS $0.178 \pm 0.265$, CON $0.068 \pm 0.061$ ).

In the chi-square test, 2 sleep-related words and 14 neutral words elicited significantly different responses between the two groups regarding whether the words were related to sleep (Table 1). Compared to the CON group, the INS group responded more frequently that the 14 neutral words were sleep-related (eggplant $\chi^{2}=5.676$, mackerel $\chi^{2}=4.421$, carrot $\chi^{2}=5.401$, mountain climbing boots $\chi^{2}=$ 5.964, stationery case $\chi^{2}=4.421$, Chinese cabbage $\chi^{2}=5.676$, soap $\chi^{2}=4.286$, watermelon $\chi^{2}=5.979$, table $\chi^{2}=5.676$, key $\chi^{2}=4.654$, cucumber $\chi^{2}=4.654$, citron $\chi^{2}=5.676$, carnation $\chi^{2}=4.421$, bean sprouts $\left.\chi^{2}=5.676 ; \mathrm{p}<0.05\right)$. For sleep-related words, the INS group was less likely to categorize them as sleep-related words than the CON group (rouse $\chi^{2}=4.210$, sleepwalker $\chi^{2}=4.421 ; \mathrm{p}<0.05$ ).

\section{DISCUSSION}

In the current study, we identified sleep-related attentional bias to word stimuli. We identified the bias in two different manners: subjective emotional valence and subjective perception about whether the word is associated with sleep. There were two main findings. First, there was no difference in emotional valence based on the group and type of word. Second, insomnia patients tended to perceive neutral words as sleep-related words even though they did not rate the valence of neutral words differently than sleep-related words.

Our results show that sleep-related attentional bias existed for neutral word stimuli rather than sleep-related word stimuli. Similar patterns of interpretive bias to neutral stimuli have been reported in previous studies. Ree et al. [25] showed that poor sleepers interpret ambiguous scenarios as threatening more often than normal sleepers. Akram et al. [26] showed that insomnia patients perceived their own faces as more tired than neutral, while normal sleepers perceived their faces as more alert. Beattie et al. [27] compared eye movements toward sleep-related items in natural scenes between individuals with insomnia symptoms and normal sleepers. Individuals with insomnia symptoms fixated longer on bed regions in complex natural scenes, even though there was no significant difference in attention to specific sleep-related items.

This interpretive bias supports distorted perception from obsessive monitoring in Harvey's cognitive model [13]. Interpretive bias toward neutral stimuli commonly occurs in the bedroom, especially during the pre-sleep period [28]. Insomnia patients selectively attend to internal and external cues that can be a subjective sign to promote or prevent falling asleep. For example, noises such 
Table 1. Differences in sleep-related words between participants

\begin{tabular}{|c|c|c|c|c|c|c|}
\hline \multirow{2}{*}{ Type of word } & \multirow{2}{*}{ Words in Korean } & \multirow{2}{*}{ Words in English } & \multirow{2}{*}{$\chi^{2}$} & \multicolumn{2}{|c|}{ Percentage of sleep-related response (\%) } & \multirow{2}{*}{$\mathrm{p}$ value } \\
\hline & & & & Insomnia patient & Healthy control & \\
\hline \multirow{2}{*}{ Sleep-related } & 깨우다 & Rouse & 4.210 & 81.8 & 100.0 & $<0.05$ \\
\hline & 몽유병 & Sleepwalker & 4.421 & 81.0 & 100.0 & \\
\hline \multirow[t]{14}{*}{ Neutral } & 가지 & Eggplant & 5.676 & 23.8 & 0.0 & $<0.05$ \\
\hline & 고등어 & Mackerel & 4.421 & 19.0 & 0.0 & \\
\hline & 당근 & Carrot & 5.401 & 22.7 & 0.0 & \\
\hline & 등산화 & Mountain climbing boots & 5.964 & 35.0 & 4.8 & \\
\hline & 문갑 & Stationery case & 4.421 & 19.0 & 0.0 & \\
\hline & 배추 & Chinese cabbage & 5.676 & 23.8 & 0.0 & \\
\hline & 비누 & Soap & 4.286 & 28.6 & 4.8 & \\
\hline & 수박 & Watermelon & 5.979 & 25.0 & 0.0 & \\
\hline & 식탁 & Table & 5.676 & 23.8 & 0.0 & \\
\hline & 열쇠 & Key & 4.654 & 20.0 & 0.0 & \\
\hline & 오이 & Cucumber & 4.654 & 20.0 & 0.0 & \\
\hline & 유자 & Citron & 5.676 & 23.8 & 0.0 & \\
\hline & 카네이션 & Carnation & 4.421 & 19.0 & 0.0 & \\
\hline & 콩나물 & Bean sprouts & 5.676 & 23.8 & 0.0 & \\
\hline
\end{tabular}

as a neighbor arriving home can be a cue to inhibit sleep, while the feeling of a slowing heart rate can be a cue to promote sleep. This interpretive bias reinforces the negative cognitive activity, such as excessive worry, and results in repetition of the cognitive cycle in Harvey's model.

Another possible role of interpretive bias is that it can create an association between the neutral bedroom space and a negative affect (e.g., arousal state). If insomnia patients are exposed to repeated stressful situations, such as perceiving noises as cues to prevent falling asleep or staying awake all night in a bedroom, their own bedrooms will trigger stress as a conditioned response. For this reason, insomnia patients often sleep better in a space other than their own bedroom. Stimulus control, such as using the bed only for sleeping and leaving the bedroom if patients do not fall asleep in 20 to 30 minutes, can facilitate sleep initiation [29].

There are some limitations to our study. First, our study was a preliminary report, so we could analyze only small samples. Second, a study of more types of words such as negative valence words or positive valence words would be required to better understand the effect of mood on interpretive bias. Third, we could not identify the difference in attentional bias based on the severity of psychiatric symptoms such as insomnia, depression, or anxiety in this report. State anxiety and insomnia duration can be major factors underlying attentional bias in insomnia. Indeed, state anxiety underlies the conditioned hyperarousal seen in patients with insomnia, and longer periods of insomnia increase attentional bias toward sleep-related stimuli.

In conclusion, we identified a sleep-related attentional bias toward neutral words among insomnia patients. These patients perceived neutral words as sleep-related words more often than normal controls, even though they did not have biased emotional valence to neutral words.

\section{Acknowledgments}

This research was supported by the Basic Science Research Program of the National Research Foundation of Korea funded by the Ministry of Science, ICT, and Future planning (no. NRF-2015 R1C1A2A01054060, NRF-2018R1D1A1B07049704).

\section{Conflicts of Interest}

The authors have no potential conflicts of interest to disclose.

\section{Author Contributions}

Conceptualization: Yu Jin Lee. Data curation: Soohyun Kim, Seong min Oh, Jeong Eun Jeon, Ha Young Lee, Inkyung Park, Juhyun Park, Mi hyun Lee. Formal analysis: Soohyun Kim. Funding acquisition: Yu Jin Lee. Investigation: Soohyun Kim, Seong min Oh, Jeong Eun Jeon, Ha Young Lee, Inkyung Park, Juhyun Park, Mi hyun Lee. Project administration: Yu Jin Lee. Resources: Yu Jin Lee. Supervision: Yu Jin Lee. Visualization: Soohyun Kim. Writing_original draft: Soohyun Kim. Writing—review \& editing: Soohyun Kim, Yu Jin Lee.

\section{ORCID iDs}

Yu Jin Lee (D)

https://orcid.org/0000-0001-5195-2579

Soohyun Kim (D)

https://orcid.org/0000-0003-0424-8575

\section{REFERENCES}

1. Bhaskar S, Hemavathy D, Prasad S. Prevalence of chronic insomnia in adult patients and its correlation with medical comorbidities. J Family Med Prim Care 2016;5:780-784.

2. American Psychiatric Association. Diagnostic and statistical manual of mental disorders: DSM-5. 5th ed. Arlington (VA): American Psychiatric Publishing, Inc.; 2013. xliv, 947-xliv, p.352.

3. Fortier-Brochu E, Beaulieu-Bonneau S, Ivers H, Morin CM. Insomnia and 
daytime cognitive performance: a meta-analysis. Sleep Med Rev 2012;16: 83-94.

4. Baglioni C, Battagliese G, Feige B, Spiegelhalder K, Nissen C, Voderholzer U, et al. Insomnia as a predictor of depression: a meta-analytic evaluation of longitudinal epidemiological studies. J Affect Disord 2011;135:10-19.

5. Taylor DJ, Lichstein KL, Durrence HH, Reidel BW, Bush AJ. Epidemiology of insomnia, depression, and anxiety. Sleep 2005;28:1457-1464.

6. Vgontzas AN, Liao D, Bixler EO, Chrousos GP, Vela-Bueno A. Insomnia with objective short sleep duration is associated with a high risk for hypertension. Sleep 2009;32:491-497.

7. Suka M, Yoshida K, Sugimori H. Persistent insomnia is a predictor of hypertension in Japanese male workers. J Occup Health 2003;45:344-350.

8. Vgontzas AN, Liao D, Pejovic S, Calhoun S, Karataraki M, Bixler EO. Insomnia with objective short sleep duration is associated with type 2 diabetes: a population-based study. Diabetes Care 2009;32:1980-1985.

9. Mallon L, Broman JE, Hetta J. High incidence of diabetes in men with sleep complaints or short sleep duration: a 12-year follow-up study of a middleaged population. Diabetes Care 2005;28:2762-2767.

10. Carney CE, Edinger JD, Morin CM, Manber R, Rybarczyk B, Stepanski EJ, et al. Examining maladaptive beliefs about sleep across insomnia patient groups. J Psychosom Res 2010;68:57-65.

11. Schutte-Rodin S, Broch L, Buysse D, Dorsey C, Sateia M. Clinical guideline for the evaluation and management of chronic insomnia in adults. J Clin Sleep Med 2008;4:487-504.

12. Harvey AG, Tang NK. (Mis)perception of sleep in insomnia: a puzzle and a resolution. Psychol Bull 2012;138:77-101.

13. Harvey AG. A cognitive model of insomnia. Behav Res Ther 2002;40:869893.

14. Harris K, Spiegelhalder K, Espie CA, MacMahon KM, Woods HC, Kyle SD. Sleep-related attentional bias in insomnia: a state-of-the-science review. Clin Psychol Rev 2015;42:16-27.

15. Spiegelhalder K, Espie C, Nissen C, Riemann D. Sleep-related attentional bias in patients with primary insomnia compared with sleep experts and healthy controls. J Sleep Res 2008;17:191-196.

16. Spiegelhalder K, Kyle SD, Feige B, Prem M, Nissen C, Espie CA, et al. The impact of sleep-related attentional bias on polysomnographically measured sleep in primary insomnia. Sleep 2010;33:107-112.

17. Taylor LM, Espie CA, White CA. Attentional bias in people with acute versus persistent insomnia secondary to cancer. Behav Sleep Med 2003;1:200-212.

18. Lundh LG, Froding A, Gyllenhammar L, Broman JE, Hetta J. Cognitive bias and memory performance in patients with persistent insomnia. Scand J Behav Ther 1997;26:27-35.

19. Sagaspe P, Sanchez-Ortuno M, Charles A, Taillard J, Valtat C, Bioulac B, et al. Effects of sleep deprivation on Color-Word, Emotional, and Specific Stroop interference and on self-reported anxiety. Brain Cogn 2006;60:76-87.

20. Spiegelhalder K, Baglioni C, Regen W, Kyle SD, Nissen C, Hennig J, et al. Brain reactivity and selective attention to sleep-related words in patients with chronic insomnia. Behav Sleep Med 2018;16:587-600.

21. Barclay NL, Ellis JG. Sleep-related attentional bias in poor versus good sleepers is independent of affective valence. J Sleep Res 2013;22:414-421.

22. Zhou N, Zhao C, Yang T, Du S, Yu M, Shen H. Attentional bias towards sleeprelated stimuli in insomnia disorder: a behavioural and ERP study. J Sleep Res 2018;27:e12652.

23. Spiegelhalder K, Espie C, Riemann D. Is sleep-related attentional bias due to sleepiness or sleeplessness? Cogn Emot 2009;23:541-550.

24. Kim HS. Survey on the use frequency of modern Korean 2, vol. 2. Seoul: National Institute of Korean Language; 2005.

25. Ree MJ, Pollitt A, Harvey AG. An investigation of interpretive bias in insomnia: an analog study comparing normal and poor sleepers. Sleep 2006;29:13591362.

26. Akram U, Ellis JG, Myachykov A, Barclay NL. Misperception of tiredness in young adults with insomnia. J Sleep Res 2016;25:466-474.

27. Beattie L, Bindemann M, Kyle SD, Biello SM. Attention to beds in natural scenes by observers with insomnia symptoms. Behav Res Ther 2017;92:5156.

28. Robertson JA, Broomfield NM, Espie CA. Prospective comparison of subjective arousal during the pre-sleep period in primary sleep-onset insomnia and normal sleepers. J Sleep Res 2007;16:230-238.

29. Zwart CA, Lisman SA. Analysis of stimulus control treatment of sleep-onset insomnia. J Consult Clin Psychol 1979;47:113-118. 